\title{
Announcement: Corrections in Physical Review Publications
}

The Physical Review journals and Reviews of Modern Physics now make Corrections of minor errors in published papers. This will end the need for follow-up publication of Publisher's Notes and many Errata across the Physical Review family. We will continue to publish Errata for issues that are too involved to be addressed as Corrections and/or have significant impact on the scientific content of the paper.

We will indicate Corrections on the paper's web page and include the dates. The same information will appear at the end of the PDF version, following the references. If timing allows, we will correct the print version, but we will not correct a printed article after it appears in a print issue. Please note that we will not use Corrections to update information that changes following publication, such as author contact information, publication of references, etc. We aim only to ensure that a paper's content accurately reflects the information that was available when the paper was published.

The Physical Review journals and Reviews of Modern Physics have also partnered with the Crossmark version of record metadata service. Crossmark ensures that the version of a published paper at hand is the most recent and provides additional information about the paper. We hope that contributors and readers find both Corrections and Crossmark to be useful.

Published 3 January 2018

DOI: 10.1103/PhysRevAccelBeams.21.010002 